Objectives: To investigate the effect of macrophage conditioned media on fibroblast activation.

Methods: PBMC-derived macrophages from healthy control $(n=3$ females, mean age $50.8 \pm 21.9$ years) and diffuse scleroderma $(n=4$ females, mean age $54.8 \pm 15.7$ years, mean disease duration $73.2 \pm 90.3$ months, 2 with antiScl70 and 2 with antiRNA polymerase antibodies) individuals were cultured in RPMI/10\% FBS/MCSF $(4 \mathrm{ng} / \mathrm{ml}) / \mathrm{P} / \mathrm{S}$, quiesced in media with $1 \% \mathrm{BSA}$ replacing the $\mathrm{FBS}$, and left untreated (M0) or treated with IL-6 $(50 \mathrm{ng} / \mathrm{ml}, \mathrm{M}(\mathrm{IL}-6))$ for 24 hours. The cultures were replaced with fresh media and collected after 24 hours. Conditioned media were applied to healthy control skin fibroblasts (24 hours) and fibroblast expression of fibrotic proteins was assessed by Western Blot, using $\beta$-tublin and TBP as loading controls. As control, fibroblasts from healthy volunteers were left untreated by culturing in non-conditioned media (fresh RPMI/ $1 \% \mathrm{BSA} / \mathrm{M}-\mathrm{CSF}$ $(4 \mathrm{ng} / \mathrm{ml}) / \mathrm{P} / \mathrm{S})$.

Results: Fibroblast expression of collagen type I and connective tissue growth factor (CTGF) were not significantly different between untreated and macrophage conditioned medium treatment groups. Baseline levels of collagen type I were high in the fibroblasts cultured in non-conditioned media, and there was a trend towards increased CTGF expression in all conditioned media-treated groups compared to untreated fibroblasts in non-conditioned media. A 2.6-fold increase in $\alpha$-smooth muscle actin ( $\alpha$-SMA) was observed in the healthy control M (IL-6)conditioned medium-treated group compared to the group of fibroblasts cultured in non-conditioned media (one-way ANOVA with Sidak multiple comparison, $\mathrm{p}=0.048$ ).

Conclusions: After 24 hours treatment, control dermal fibroblasts treated with media of IL-6-polarised healthy control macrophages expressed higher levels of $\alpha$-SMA compared to fibroblasts cultured in non-conditioned medium. A trend towards increased CTGF was also observed. These results suggest that paracrine factors in the IL-6-activated macrophage secretome may promote differentiation of fibroblasts into myofibroblasts, which is a key component of wound healing and scleroderma fibrosis.

References:

[1] Fuschiotti P. Current perspectives on the immunopathogenesis of systemic sclerosis. Immunotargets Ther. 2016;5:21-35.

[2] Denton CP, Shi-Wen X, Sutton A, et al. Scleroderma fibroblasts promote migration of mononuclear leucocytes across endothelial cell monolayers. Clin Exp Immunol. 1998;114:293-300.

[3] Khan K, Xu S, Nihtyanova S, et al. Clinical and pathological significance of interleukin 6 overexpression in systemic sclerosis. Ann Rheum Dis. 2012;71:1235-42.

Disclosure of Interest: None declared

DOI: 10.1136/annrheumdis-2017-eular.4868

\section{AB0167 CALCIUM INFLUX KINETICS AND THE CHARACTERISTICS OF POTASSIUM CHANNELS IN PERIPHERAL T LYMPHOCYTES IN SYSTEMIC SCLEROSIS}

N. Legány ${ }^{1}$, G. Toldi ${ }^{2}$, I. Ocsovszki ${ }^{3}$, L. Kovács ${ }^{1}$, A. Balog ${ }^{1} .{ }^{1}$ Department of Rheumatology and Immunology, Faculty of Medicine, Albert Szent-Györgyi Health Center, University of Szeged, Szeged; ${ }^{2}$ First Department of Obstetrics and Gynecology, Semmelweis University, Budapest; ${ }^{3}$ Department of Biochemistry, Faculty of Medicine, Albert Szent-Györgyi Health Center, University of Szeged, Szeged, Hungary

Background: Systemic sclerosis (SSc) is a chronic connective tissue disorder characterized by microvascular injury, fibrosis and autoimmunity that affects the skin and internal organs. The short-term activation of peripheral blood T lymphocytes plays a crucial role in initiating and maintaining the chronic inflammation. The transient increase of the cytoplasmic free calcium level plays a key role in the process of lymphocyte activation. Kv1.3 and IKCa1 potassium channels are important regulators of the maintenance of calcium influx during lymphocyte activation. The influx of calcium is maintained by the function of potassium channels that conserve the electrochemical potential gradient via the efflux of potassium from the cytoplasm. Recent reports raised the notion that the inhibition of lymphocyte potassium channels, especially that of the Kv1.3 channel would be an straightforward solution for specific immunosuppression in autoimmune disorders. Furthermore, our previous studies described an alteration of the short-term activation of peripheral lymphocytes in rheumatoid arthritis and primary Sjögren's syndrome (pSS), and the overexpression of Kv1.3 channels in pSS.

Objectives: Therefore, in this study we aimed to characterize the effects of lymphocyte potassium channel inhibition on short-term peripheral blood T lymphocyte activation in major lymphocyte subsets in SSc.

Methods: We enrolled 12 healthy individuals and 16 SSc patients. We evaluated calcium influx kinetics following activation in CD4, Th1, Th2 and CD8 cells applying a novel kinetic flow cytometry approach. We assessed the sensitivity of the above subsets to specific inhibition of the Kv1.3 and IKCa1 potassium channels. We also assessed the Kv1.3 expression on lymphocytes.

Results: We observed increased parameters of calcium influx in CD8+ lymphocytes' as compared with Th1 cells in SSc. However, the activation of CD8+ cells was lower in SSc compared to healthy controls. Moreover, activation of Th1 lymphocytes was slower in SSc than in healthy controls. The inhibition of IKCa1 potassium channel decreased the activation of CD8+ lymphocytes in healthy controls and the activation of Th1 cells in SSc. The inhibition of Kv1.3 channel modified the dynamics of activation of Th1 and Th2 lymphocytes in SSc.

Conclusions: The altered function of CD8+ cells and the specific inhibition of potassium channels seem to be a consequence of altered calcium influx kinetics in SSc, distinguishing it both from healthy controls and other autoimmune diseases. Acknowledgements: Attila Balog and Gergely Toldi were supported by the János Bolyai Scholarship. Gergely Toldi is an International Society for the Advanecement of Cytometry (ISAC) Scholar.

Disclosure of Interest: None declared

DOI: 10.1136/annrheumdis-2017-eular.5310

\section{AB0168 PROTECTIVE EFFECTS OF EPIGALLOCATECHIN 3 GALLATE ON FIBROSIS IN SCLERODERMA MODEL}

A. Kocak $^{1}$, D. Harmancı ${ }^{1}$, M. Birlik ${ }^{2}$, S. Sarioglu ${ }^{3}$, G. Guner Akdogan ${ }^{4}$ ${ }^{1}$ Department of Molecular Medicine, Institute of Health Sciences, Dokuz Eylul University; ${ }^{2}$ Department of Internal Medicine, Division of Rheumatology \& Immunology; ${ }^{3}$ Department of Medical Pathology, School of Medicine, Dokuz Eylul University; ${ }^{4}$ Department of Biochemistry, School of Medicine, Izmir University of Economics, Izmir, Turkey

Background: Scleroderma (SSc) is a disease that shows involvement in internal organs or on the skin characterized by fibrosis (1). Dermis thickening and uncontrolled extracellular matrix (ECM) increase are seen in this disease whose pathogenesis is not fully understood. TGF- $\beta /$ Smad $2 \& 3$ pathway is pivotal role in SSc pathogenesis via induction of profibrotic molecules including collagen and by decrease of matrix metalloproteinases (MMPs) synthesis $(2,3)$. The occurrence of the myofibroblast phenotype at fibrosis is thought to be responsible for the contracted regions of the affected tissues (4).

Objectives: The aim of this study with bleomycin (BLM) formed in an experimental model of scleroderma is to investigate the potential effects of epigallocatechin-3gallate (EGCG) against fibrosis.

Methods: $32 \mathrm{Balb} / \mathrm{c}$ female mice were randomly selected into four groups.For 21 days: (1) Control group (n: 8) was given $100 \mu \mathrm{L}$ subcutan (sc) saline (SF) once a day, $100 \mu \mathrm{L}$ intraperitoneal (ip) SF twice a week, (2) BLM group (n: 8) was given $100 \mu \mathrm{L}$ (100 ug) sc BLM once a day, $100 \mu \mathrm{L}$ ip SF twice a week, (3) BLM + EGCG group (n:8) was given $100 \mu \mathrm{L}$ (100 ug) sc BLM once a day, $100 \mu \mathrm{L}(100 \mu \mathrm{g})$ ip EGCG twice a week, (4) EGCG group (n: 8) was given $100 \mu \mathrm{L} s c$ SF once a day, $100 \mathrm{~mL}(100 \mu \mathrm{g})$ ip ECCG twice a week. Hematoxylin\&eosin and Masson trichrome staining of dermal areas were performed. Myofibroblast activity was measured using alpha smooth muscle actin antibody ( $\alpha \mathrm{SMA}$ ) by immunohistochemistry. Expression levels of MMP-1, MMP8 , MMP-13 and P-SMAD protein were examined by western blot. Expression levels of TGF- $\beta$ mRNA were examined by qPCR. All of the statistical analyses were performed using SPSS software and the quantitative data were expressed as the means \pm SEM.The quantitative variables were compared using the a ANOVA-Sidak. Statistical significance was defined as $p<0.05$
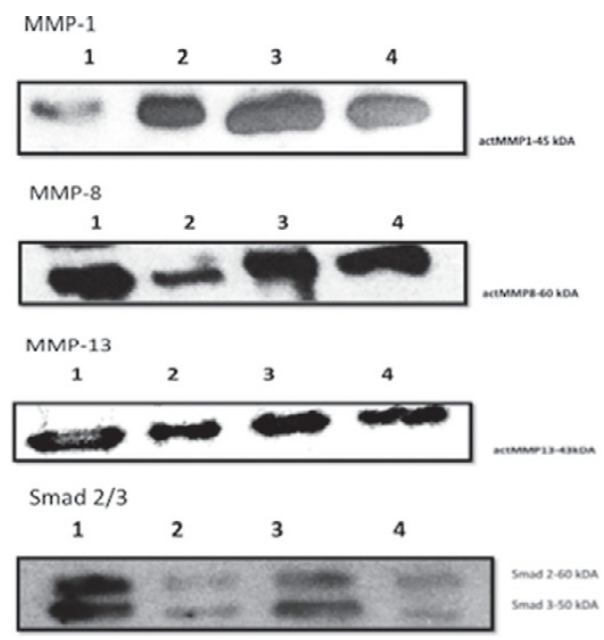

P-smad 2/3

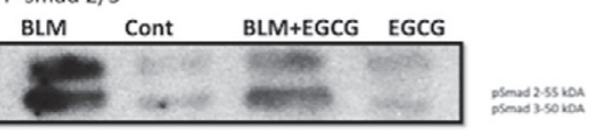

B-Actin

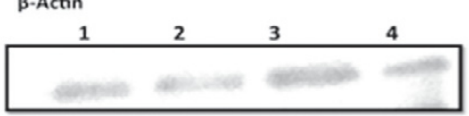

Figure 5. MMP-1, MMP-8, MMP-13, SMAD $2 / 3$ and p-SMAD2/3 expression $\beta$ - Actin used for internal control. 
Results: When compared to sham,control and experimental groups was observed to have reduced connective tissue fibrosis in dermis area, according to Masson Trichrome results. EGCG group showed a significant reduction in fibrosis at the dermal surface area with respect to hematoxylin measurements. MMP-1, MMP-8, p-SMAD 2/3 protein levels and TGF- $\beta$ mRNA expression were slightly decreased in EGCG Group compared with the other tested groups $(p<0.05)$. Otherwise, MMP-13 wasn't change between groups.

Conclusions: This study contributes to the potential use of EGCG as a treatment for fibrosis in SSc patients. Also, MMP-1\&MMP-8 may play an important role in the etiology of SSc.

\section{References:}

[1] Varga, J. 2012. 15. Scleroderma-from pathogenesis to comprehensive management.Varga, J., Denton, C., Wigley, F.M./Denton C. New York: Springer.

[2] Ihn H. 2008. Autocrine TGF-beta signaling in the pathogenesis of systemic sclerosis. J Dermatol Sci 49: 103-113.

[3] Derk CT. 2007. Transforming growth factor-beta (TGF-beta) and its role in the pathogenesis of systemic sclerosis: a novel target for therapy? Recent Pat Inflamm Allergy Drug Discov 1: 142-145.

[4] Desmouliere A, Chaponnier C, Gabbiani G. 2005. Tissue repair, contraction, and the myofibroblast. Wound Repair Regen. 13(1):7-12.

Acknowledgements: This research was supported by a grant supplied from "Dokuz Eylul University Research Fund" and carried out at Dokuz Eylul University Medicine Faculty of Research Laboratory (R-LAB).

Disclosure of Interest: None declared

DOI: 10.1136/annrheumdis-2017-eular.2076

\section{AB0169 EVALUATION OF FREQUENCY AND TYPE OF PHYSICAL THERAPY IN MORE THAN 3400 PATIENTS WITH SYSTEMIC SCLEROSIS}

D. Belz ${ }^{1}$, P. Moinzadeh ${ }^{1}$, N. Blank ${ }^{2}$, E. Siegert ${ }^{3}$, J. Henes ${ }^{4}$, M. Worm ${ }^{5}$, C. Sunderkoetter ${ }^{6}$, M. Schmalzing ${ }^{7}$, A. Kreuter ${ }^{8}$, C. Gunther ${ }^{9}$, L. Susok ${ }^{10}$, G. Zeidler ${ }^{11}$, I. Koetter ${ }^{12}$, U. Mueller-Ladner ${ }^{13}$, T. Krieg ${ }^{1}$, A. Juche ${ }^{14}$, T. Schmeiser ${ }^{15}$, G. Riemekasten ${ }^{16}$, E. Aberer ${ }^{17}$, N. Gaebelein-Wissing ${ }^{18}$, J.H.W. Distler ${ }^{19}$, M. Sárdy ${ }^{20}$, C. Pfeiffer ${ }^{21}$, K. Kuhr ${ }^{22}$, N. Hunzelmann ${ }^{1}$. ${ }^{1}$ Dermatology, University Hospital Cologne, Cologne; ${ }^{2}$ Rheumatology, University Hospital Heidelberg, Heidelberg; ${ }^{3}$ Rheumatology, Charité Universitätsmedizin Berlin, Berlin; ${ }^{4}$ Rheumatology, University Hospital Tuebingen, Tuebingen; ${ }^{5}$ Dermatology, Charité Universitätsmedizin Berlin, Berlin; ${ }^{6}$ Dermatology, University Hospital Muenster, Muenster; ${ }^{7}$ Rheumatology, University Hospital Wuerzburg, Wuerzburg; ${ }^{8}$ Dermatology, HELIOS St. Elisabeth Klinik Oberhausen, Oberhausen; ${ }^{9}$ Dermatology, University Hospital Carl Gustav Carus, Dresden; ${ }^{10}$ Dermatology, St. Josef Hospital Bochum, Bochum; ${ }^{11}$ Rheumatology, Johanniter-Krankenhaus im Fläming Treuenbrietzen, Treuenbrietzen;

${ }^{12}$ Rheumatology, Asklepios Klinik Altona, Hamburg; ${ }^{13}$ Rheumatology, Justus Liebig University Giessen, Kerckhoff Clinic, Bad Nauheim; ${ }^{14}$ Immanuel Krankenhaus Berlin-Buch, Berlin; ${ }^{15}$ Rheumatology, Krankenhaus St. Josef, Wuppertal; ${ }^{16}$ Rheumatology, University Medical Center-UKSH, Luebeck, Germany; ${ }^{17}$ Dermatology, Medical University of Graz, Graz, Austria; ${ }^{18}$ Dermatology, HELIOS University Hospital Wuppertal, Wuppertal;

${ }^{19}$ Rheumatology, University Hospital Erlangen, Erlangen; ${ }^{20}$ Dermatology, Ludwig Maximilians University Hospital, Munich; ${ }^{21}$ Dermatology, University Medical Center Ulm, UIm; ${ }^{22}$ IMSIE, University of Cologne, Cologne, Germany

Background: Systemic sclerosis (SSc) is a chronic fibrosing autoimmune disease which leads to severe musculoskeletal dysfunction, disability and contractures. Little is known on the type and extent of physical therapy (PT) prescribed to SSc patients in daily practice.

Objectives: To determine the type and frequency of PT received by SSc patients. Methods: The data of 3430 clinically well defined SSc patients registered in the database of the German Network for Systemic Sclerosis were analyzed using SPSS.

Results: $48,5 \%(1662 / 3430)$ of the patients were treated with PT. The most frequently used form of PT was lymphatic drainage $(23,6 \% / 876)$, followed by physical exercise therapy $(22 \% / 817)$ and paraffin wax bath (10,5\%/389). About half of the patients $(46,9 \%)$ received two or three different forms of PT simultaneously. The prescription of PT did not correlate with the SSc subtype, as 49,5\% (503/1016) of dcSSc patients, 50,3\% (850/1689) of IcSSc patients and 45,7\% (143/313) of SSc-Overlap patients received PT. PT was significantly more often prescribed to patients with pulmonary fibrosis in $51,1 \%(617 / 1208)$, synovitis in $61,6 \%(299 / 485)$ and CK elevation in $61,1 \%(174 / 285)(p=0,001-0,029)$. PT did not correlate with the extent of skin fibrosis as measured by mRSS. Interestingly, patients with joint contractures $(45,5 \%)(388 / 853)$ or tendon friction rubs $(40,6 \%)(114 / 281)$ received significantly less often PT $(p=0,006 / 0,045)$. Comparing the prescription of PT during the initial period $2003-2008(49,1 \% ; 1937 / 3942)$ with the follow up period 2009-2013 (45,3\%; 2217/4899), a significant decrease of PT prescription was observed $(p<0,001)$. Patterns of PT prescription differed significantly between medical subspecialties $(p<0,005)$ i.e. rheumatologists, dermatologists.

Conclusions: Although SSc is characterized by considerable disability and restriction of motion, less than $50 \%$ of patients received PT. The significant decrease in PT prescription during recent years may reflect lack of knowledge how to prescribe PT and more restrictive insurance regulations.

Disclosure of Interest: None declared
DOI: 10.1136/annrheumdis-2017-eular.3361

\section{AB0170 DIMINISHED PERIPHERAL T-CELL ACTIVATION ALONG WITH MARKED TH17 CIRCULATING PROFILE IN SSC}

E. Krasimirova ${ }^{1}$, D. Kalinova ${ }^{2}$, T. Velikova ${ }^{1}$, E. Ivanova-Todorova ${ }^{1}$,

K. Tumangelova-Yuzeir ${ }^{1}$, R. Rashkov ${ }^{2}$, D. Kyurkchiev ${ }^{1} .{ }^{1}$ Laboratory of Clinical immunology; ${ }^{2}$ Clinic of Rheumatology, University Hospital St. Ivan Rilski, Medical University of Sofia, Sofia, Bulgaria

Background: Systemic sclerosis (SSc) is a rare, debilitating connective tissue disease characterized by immunological alterations, vasculopathy and progressive skin and multiorgan fibrosis. The autoimmune dysregulation in SSc comprises lymphocyte activation that leads to autoantibody production, abnormal production of cytokines and chemokines, and impairment of the innate immunity. Recent research has shown that the T cell activation and especially $T$ helper cells play an important role in the pathogenesis of SSc. Amongst them are the proinflammatory Th17 cells.

Objectives: To investigate T-cell activation, the percentage of Th17 cells and the circulating cytokine profile in SSc.

Methods: We enrolled a total of 24 SSc patients and 16 healthy controls in the study and divided the patients as having diffuse cutaneous SSc (dcSSc, $n=13$ ) or limited cutaneous SSc (IcSSc, $n=11$ ). Peripheral venous blood samples were collected from all subjects. We examined the percentage of activated $T$ cells (unstimulated and upon stimulation with PHA-M) and of Th17 cells by flow cytometry in both patients and controls. We used ELISA to quantitate the serum levels of human IL-6, TGF- $\beta 1$, and IL-17A.

Results: We identified a decreased percentage of activated T cells (CD3+CD69+) in PHA-stimulated samples from SSc patients in comparison with healthy controls, $p<0.001$. However, we did not establish a correlation between the down-regulated CD3+CD69+ cells and the SSc phenotype.

With regard to Th17 cells, our patients demonstrated increased percentage as opposed to controls, $p=0.031$. We detected up-regulated Th17 cells within the IcSSc subset against controls, $p=0.025$. However, no difference was found between dcSSc and IcSSc patients. Regarding the peripheral cytokine profile, we detected raised levels of IL- $6, p<0.001$, TGF- $\beta 1, p=0.02$, and IL-17A, $p<0.001$ in patients when compared to controls. Furthermore, we found increased circulating TGF- $\beta$, IL- 6 and IL-17A in the ICSSc subset versus healthy individuals, as it follows TGF- $\beta 1, p=0.031$, IL- $6, p<0.001$, and IL-17A, $p<0.001$. Furthermore, circulating IL-17A was higher in ICSSc as opposed to dcSSc subset, $p=0.008$. Within the dcSSc phenotype, we detected raised levels of IL-17A and IL-6 versus controls: IL-17A, $p<0.001$, IL- $6, p<0.001$.

Conclusions: Our results demonstrate down-regulated T-cell activation upon PHA-stimulation along with pronounced peripheral Th17 profile, and related cytokines in SSc patients, suggesting their implication in the pathogenesis of SSc. References:

[1] Brembilla NC, Chizzolini C. T cell abnormalities in systemic sclerosis with a focus on Th17 cells. Eur Cytokine Netw 2012; 23 (4): 128-139.

[2] Raja J, Denton CP. Cytokines in immunopathology of systemic sclerosis. Semin Immunopathol 2015; 37 (5): 543-557.

Disclosure of Interest: None declared

DOI: 10.1136/annrheumdis-2017-eular.5767

\section{AB0171 ANTI-CARBAMYLATED PROTEIN ANTIBODIES IN PATIENTS WITH SYSTEMIC SCLEROSIS: AN INTRIGUING ASSOCIATION WITH SKIN INVOLVEMENT}

E. Favoino $^{1}$, M. Prete $^{1}$, S. Vettori ${ }^{2}$, A. Corrado ${ }^{3}$, F.P. Cantatore ${ }^{3}$, G. Valentini ${ }^{2}$, F. Perosa ${ }^{1} .{ }^{1}$ Department of Biomedical Sciences and Human Oncology, Systemic Rheumatic and Autoimmune Disease Unit, University of Bari Medical School, Bari; ${ }^{2}$ Department of Clinical and Experimental Internal Medicine, Rheumatology Section, Second University of Naples, Naples; ${ }^{3}$ Department of Medical and Surgery Sciences, Rheumatology Unit, University of Foggia, Foggia, Italy

Background: Systemic sclerosis (SSc), one of the most complex connective tissue disease, is characterized by three pathogenic events namely, vascular damage, autoimmunity and fibroblast activation, leading to a widespread fibrosis of skin and internal organs $(1,2)$. Previous studies showed that 1) carbamylation mainly affects structural proteins undergoing to a low turn-over rate, namely dermal skin and tendons-associated proteins; and that 2) carbamylated proteins accumulate in skin in an age-dependent manner, contributing to tissue alteration (3).

Objectives: As dermis is a disease target and anti-carbamylated protein antibodies (anti-CarP Ab) have been reported in patients with SSc (4), we sought to evaluate any relationship between anti-CarP Ab and clinical parameters reflecting skin involvement in SSc.

Methods: Serum samples and clinical data from 123 patients with SSc were collected. Anti-CarP Ab were detected by indirect ELISA, using carbamylated bovine serum albumin as the antigen. Serum Anti-CarP Ab levels were also measured in 41 healthy aged-matched individuals. Clinical data were retrieved as previously reported (5).

Results: The mean serum levels of anti-CarP Ab did not statistically differ 